\title{
Retraction: In Situ Control of Diamagnetism by Electric Current in $\mathrm{Ca}_{3}\left(\mathbf{R u}_{1-x} \mathbf{T i}_{x}\right)_{2} \mathbf{O}_{7}$ [Phys. Rev. Lett. 122, 196602 (2019)]
}

Chanchal Sow $\odot$, Ryo Numasaki, Giordano Mattoni, Shingo Yonezawa, Naoki Kikugawa, Shinya Uji, and Yoshiteru Maeno

(C) (Received 25 March 2020; accepted 26 March 2020; published 23 April 2020)

DOI: $10.1103 /$ PhysRevLett.124.169902

Following the publication of the Letter, new measurements performed in Kyoto by Mattoni, Yonezawa, and Maeno [1] led to reveal a serious technical artefact that affected our published data. In particular, it became clear that a large part of the reported diamagnetic signal was arising from a mechanism that we did not anticipate. This signal can be understood as due to localized heating of the sample holder, caused by the unavoidable Joule heating in the sample.

Because the artefact affects the main experimental data, the authors unanimously agreed to retract the Letter. For the same reason, previous work on $\mathrm{Ca}_{2} \mathrm{RuO}_{4}$ [2] has also been retracted [3]. We are profoundly sorry for having overlooked this aspect, and we explain the details of this rather unexpected phenomenon in Ref. [1].

[1] G. Mattoni, S. Yonezawa, and Y. Maeno, Appl. Phys. Lett. 116, 17 (2020).

[2] C. Sow, S. Yonezawa, S. Kitamura, T. Oka, K. Kuroki, F. Nakamura, and Y. Maeno, Science 358, 1084 (2017).

[3] C. Sow et al. Science 368, 376 (2020). 